\title{
Simulating quantum field theory in curved spacetime with quantum many-body systems
}

\author{
Run-Qiu Yang $\odot,{ }^{1}$ Hui Liu, ${ }^{2}$ Shining Zhu, ${ }^{2}$ Le Luo, ${ }^{3}$ and Rong-Gen Cai ${ }^{4, *}$ \\ ${ }^{1}$ Center for Joint Quantum Studies and Department of Physics, School of Science, Tianjin University, Yaguan Road 135, Jinnan District, \\ 300350 Tianjin, People's Republic of China \\ ${ }^{2}$ National Laboratory of Solid State Microstructures and School of Physics, Collaborative Innovation Center of Advanced Microstructures, \\ Nanjing University, Nanjing, Jiangsu 210093, People's Republic of China \\ ${ }^{3}$ School of Physics and Astronomy, Sun Yat-sen University, 519082 Zhuhai, People's Republic of China \\ ${ }^{4}$ CAS Key Laboratory of Theoretical Physics, Institute of Theoretical Physics, Chinese Academy of Sciences, Beijing 100190, \\ People's Republic of China
}

(Received 14 June 2019; revised manuscript received 30 September 2019; accepted 23 March 2020; published 30 April 2020)

\begin{abstract}
This paper proposes a new general framework to build a one-to-one correspondence between quantum field theories in static $(1+1)$-dimensional curved spacetime and quantum many-body systems. We show that a massless scalar field in an arbitrary two-dimensional static spacetime is always equivalent to a site-dependent bosonic hopping model, while a massless Dirac field is equivalent to a site-dependent free Hubbard model or a site-dependent isotropic $X Y$ model. A possible experimental realization for such a correspondence in trapped-ion systems is suggested. As applications of the analog gravity model, we show that they can be used to simulate Hawking radiation of a black hole and to study its entanglement. We also show in the analog model that black holes are the most chaotic systems and the fastest scramblers in nature. We also offer a concrete example about how to get some insights about quantum many-body systems from black-hole physics.
\end{abstract}

DOI: 10.1103/PhysRevResearch.2.023107

\section{INTRODUCTION}

Quantum field theory in curved spacetime is a semiclassical approximation of quantum gravity theory, where the curved background spacetime is treated classically, while the matter fields in the curved spacetime are quantized. Although a fully successful quantum gravity theory is still not yet available, such a semiclassical approximation framework has offered us a large amount of interesting new phenomena, such as the Hawking radiation of black holes, particle production in an expanding universe, etc. (see Refs. [1,2] for some review articles). Since in general these phenomena are extremal weak, they are extremely difficult to observe in real gravity situations. Analogs of black-hole or other phenomena in curved spacetime in the laboratory offer us new perspectives on quantum effects in curved spacetime, which might help us deeply understand the nature of gravity. Following the original work of Unruh [3,4], which studies Hawking radiation in a sonic analog of a black hole, a large number of systems have been proposed and explored, such as surface waves in water flows [5], Bose-Einstein condensates (BECs) [6-8], optic systems [9-13], and ultracold atoms in optical lattices [14] (see Refs. [15-17] for reviews and references therein).

\footnotetext{
*cairg@itp.ac.cn

Published by the American Physical Society under the terms of the Creative Commons Attribution 4.0 International license. Further distribution of this work must maintain attribution to the author(s) and the published article's title, journal citation, and DOI.
}

In spite of the impressive progress that has been made theoretically and experimentally on various analog gravity systems, it is still interesting to seek some analog models which are more "pure" in theory and more easily controlled in experiment. In condensed-matter physics, there exist three basic quantum many-body models, the hopping model, the Hubbard model, and the isotropic $X Y$ model [18], which are of wide application in many fields. In this paper we find that these models also have interesting applications in quantum field theory in curved spacetime. The hopping rate in natural materials is constant; there is not enough motivation for physicists in condensed-matter physics and materials to study the "site-dependent" hopping cases. Here we do see that the site-dependent hopping cases occur in the simulation of quantum field theories in curved spacetime by quantum many-body systems.

So far most analog gravity models have focused on the simulation of Hawking radiation or other types of spontaneous particle creation, such as the Unruh effect, particle creation in the universe, and dynamical Casimir effect (see, e.g., Refs. [19-21]). Let us notice that, over the past decades, a remarkable progress in gravity and relevant fields is the proposal of anti-de Sitter (AdS)-conformal field theory (CFT) correspondence [22-24], which says that a quantum gravity in the AdS spacetime is dual to a CFT living in the AdS boundary. The connection between geometry in the bulk and entanglement entropy in the boundary is also suggested in Refs. [25,26]. Recently, based on the AdS-CFT correspondence, quantum scrambling has been suggested as a powerful tool for characterizing chaos in black holes [27,28], and Refs. [28-30] conjectured that a black hole has the 
fastest scrambling and is the most quantum chaotic system in nature.

One of the remarkable features of the AdS-CFT correspondence is the strong/weak duality: a weak gravity theory in the AdS bulk is equivalent to a strong coupled CFT in the AdS boundary. Although there exist many pieces of evidence to show the correspondence is true, it is extremely difficult, if it is not impossible, to prove the AdS-CFT correspondence. The analog gravity models provide the possibility to test experimentally the AdS-CFT correspondence.

In this paper we show that there exists a one-to-one correspondence between quantum field theories in an arbitrary two-dimensional spacetime and a site-dependent bosonic hopping model, free Hubbard model, or isotropic $X Y$ model in quantum many-body systems. As some applications of our analog gravity model, we study Hawking radiation of a black hole and its entanglement, and show that black holes are the most chaotic systems and the fastest scramblers in nature, predictions of the AdS-CFT correspondence. We also use a concrete example to show how to use the picture of blackhole physics to learn something about quantum many-body systems.

\section{QUANTUM FIELDS IN CURVED SPACETIME}

We consider a two-dimensional background spacetime with signature $(+,-)$. In the static case, the metric can always be given in the Schwarzschild coordinates $\{t, x\}$ as

$$
d s^{2}=f(x) d t^{2}-f(x)^{-1} d x^{2} .
$$

In most cases, we are interested in the static black-hole spacetime with a single nondegenerated horizon; i.e., $f(x)>0$ for $x>x_{h}$ and there is only a point at $x=x_{h}$ such that $f\left(x_{h}\right)=0$ but

$$
g_{h}=\frac{1}{2} f^{\prime}\left(x_{h}\right)>0,
$$

where $g_{h}$ is the surface gravity of the horizon, which gives the Hawking temperature $T_{H}=g_{h} /(2 \pi)$ of the black hole. The metric (1) in the coordinates $\{t, x\}$ is singular at the horizon. To overcome this shortage, we can define an infalling Eddington-Finkelstein coordinate by the coordinate transformation,

$$
t \rightarrow v, \text { such that } v=t+\int f(x)^{-1} d x .
$$

The metric (1) in the infalling Eddington-Finkelstein coordinates $\{v, x\}$ becomes

$$
d s^{2}=f d v^{2}-2 d v d x .
$$

In this case the metric no longer has the coordinate singularity at the horizon.

Let us first consider a scalar field in the two-dimensional curved spacetime. The Klein-Gordon equation of a complex scalar field in metric (3) reads

$$
m^{2} \phi-2 \partial_{v} \partial_{x} \phi-f^{\prime} \partial_{x} \phi-f \partial_{x}^{2} \phi=0 .
$$

By introducing the variable $\varphi$,

$$
m \varphi=2 \partial_{v} \phi+f \partial_{x} \phi
$$

Eq. (4) can be rewritten into two coupled first-order equations

$$
\partial_{v} \phi=-\frac{f}{2} \partial_{x} \phi+\frac{m \varphi}{2}, \quad \partial_{x} \varphi=m \phi .
$$

Now we make the variable transformation $\phi=w \sqrt{f}$ and we can rewrite the above equations into the following forms:

$$
\partial_{v} w=-\frac{f}{2} \partial_{x} w-\frac{f^{\prime}}{4} w+\frac{m \varphi}{2 \sqrt{f}}, \quad \partial_{x} \varphi=m w \sqrt{f} .
$$

In the massless limit $m \rightarrow 0$, the above two equations decouple and there is only one independent evolutional equation:

$$
\partial_{v} w=-\frac{1}{4}\left[\partial_{x}(f w)+f \partial_{x} w\right] .
$$

Eq. (7) is singular at the horizon due to $f\left(x_{h}\right)=0$. Mathematical discussions about this singularity and Eq. (8) can be found in Appendix A.

A similar result can also be obtained for Dirac field. The Dirac equation with the general vielbein $e^{\mu}{ }_{a}$ and metric $g_{\mu \nu}$ can be written as $[14,31]$

$$
i \gamma^{a} e^{\mu}{ }_{a} \partial_{\mu} \psi+\frac{i}{2} \gamma^{a} \frac{1}{\sqrt{-g}} \partial_{\mu}\left(\sqrt{-g} e_{a}^{\mu}\right) \psi-m \psi=0 .
$$

Here $g$ is the determinate of metric $g_{\mu \nu}$. The $\gamma$-matrices in the two-dimensional case are chosen such that $\gamma^{a}=\left(\sigma_{z}, i \sigma_{y}\right)$. We choose the vielbein to be

$$
e^{\mu}{ }_{a}=\left[\begin{array}{cc}
-1, & 1 \\
-\frac{f}{2}+\frac{1}{2}, & \frac{f}{2}+\frac{1}{2}
\end{array}\right]
$$

and take the decomposition

$$
\psi=\frac{1}{\sqrt{2}}\left[\begin{array}{l}
u+w \\
u-w
\end{array}\right]
$$

into account, and we find that there are two independent equations

$$
\partial_{v} w=-\frac{f}{2} \partial_{x} w-\frac{f^{\prime}}{4} w+\frac{i}{2} m u, \quad \partial_{x} u=-i m w .
$$

In the massless limit $m \rightarrow 0$, there is only one evolutional equation that remains, which is the same as Eq. (8).

\section{MAP INTO QUANTUM MANY-BODY SYSTEMS}

\section{A. Theory model}

Now let us discretize the system. The spatial position is discretized as $x=x_{n}=n d$ with $n \in \mathbb{N}$ and $d \ll \lambda_{0}$, where $\lambda_{0}$ is the effective average wavelength in the system. The functions in the fixed spacetime are then transformed into discrete forms as follows:

$$
f_{n}=f(n d), \quad w_{n}(v)=w\left(v, x_{n}\right) .
$$

The spatial derivatives in Eq. (8) are approximated by central differences. Upon a variable transformation $w_{n}=$ $(-i)^{n} e^{-i \mu v} \tilde{w}_{n}$, Eq. (8) can be rewritten into the following form:

$$
i \frac{d}{d v} \tilde{w}_{n}=-\kappa_{n} \tilde{w}_{n-1}-\kappa_{n+1} \tilde{w}_{n+1}-\mu \tilde{w}_{n}
$$


with

$$
\kappa_{n}=\frac{f_{n}+f_{n-1}}{8 d} \approx \frac{f[(n-1 / 2) d]}{4 d} .
$$

Here $\mu$ is an arbitrary constant. We see later that it can be interpreted as the chemical potential in quantum many-body systems. Due to the discretization, the discrete form is a good approximation for continuous fields if fields are slowly varying; i.e., Eq. (11) is valid in the low-energy limit.

Now let us quantize these fields themselves. This can be done by promoting field $\tilde{w}_{n}$ into an operator. For the bosonic field, we use the replacement $\tilde{w}_{n} \rightarrow \hat{a}_{n} / \sqrt{d}$ and introduce bosonic commutators such that

$$
\left[\hat{a}_{n}, \hat{a}_{m}^{\dagger}\right]=\delta_{n m},\left[\hat{a}_{n}, \hat{a}_{m}\right]=\left[\hat{a}_{n}^{\dagger}, \hat{a}_{m}^{\dagger}\right]=0 .
$$

The evolutional equation for the field operator then reads

$$
i \frac{d}{d v} \hat{a}_{n}=-\kappa_{n} \hat{a}_{n-1}-\kappa_{n+1} \hat{a}_{n+1}-\mu \hat{a}_{n} .
$$

Considering the evolutional equation in the Heisenberg picture, $i \partial_{v} \hat{a}_{n}=\left[\hat{a}_{n}, \mathcal{H}\right]$, Eq. (13) implies the following Hamiltonian:

$$
\mathcal{H}=\sum_{n}\left[-\kappa_{n}\left(\hat{a}_{n}^{\dagger} \hat{a}_{n-1}+\hat{a}_{n-1}^{\dagger} \hat{a}_{n}\right)-\mu \hat{a}_{n}^{\dagger} \hat{a}_{n}\right] .
$$

This Hamiltonian describes a bosonic hopping model and can be treated as a limit case of a certain different well-studied quantum systems. For example, in condensed-matter systems, it is the Bose-Hubbard model [32-35] with site-dependent hopping amplitude and zero on-site self-interaction.

For the Dirac field, we can do a similar thing. Taking the replacement $\tilde{w}_{n} \rightarrow \hat{c}_{n} / \sqrt{d}$ and introducing anticommutators such that

$$
\left\{\hat{c}_{n}^{\dagger}, \hat{c}_{m}\right\}=\delta_{n m},\left\{\hat{c}_{n}, \hat{c}_{m}\right\}=\left\{\hat{c}_{n}^{\dagger}, \hat{c}_{m}^{\dagger}\right\}=0,
$$

we can obtain the following Hamiltonian form:

$$
\mathcal{H}=\sum_{n}\left[-\kappa_{n}\left(\hat{c}_{n}^{\dagger} \hat{c}_{n-1}+\hat{c}_{n-1}^{\dagger} \hat{c}_{n}\right)-\mu \hat{c}_{n}^{\dagger} \hat{c}_{n}\right]
$$

This is just the free Hubbard model with site-dependent hopping. This model has been widely studied and can be realized in various different platforms (see Refs [36-38], for instance).

Hamiltonian (15) can also be rewritten into another wellstudied model in condensed-matter physics: the isotropic $X Y$ model $[39,40]$. To do that, let us introduce the following operators according to the Jordan-Wigner transformation:

$$
\sigma_{n}^{+}=\exp \left[i \pi \sum_{j=1}^{n-1} \hat{c}_{j}^{\dagger} \hat{c}_{j}\right] \hat{c}_{n}^{\dagger}, \quad \sigma_{n}^{-}=\exp \left[-i \pi \sum_{j=1}^{n-1} \hat{c}_{j}^{\dagger} \hat{c}_{j}\right] \hat{c}_{n}
$$

and $\sigma_{n}^{z}=1-2 \hat{c}_{n}^{\dagger} \hat{c}_{n}$ with the periodic-antiperiodic boundary condition. After neglecting a constant term, Hamiltonian (15) can be rewritten as

$$
\mathcal{H}=\sum_{n}\left[-\kappa_{n}\left(\sigma_{n}^{+} \sigma_{n-1}^{-}+\sigma_{n-1}^{+} \sigma_{n}\right)+\frac{1}{2} \mu \sigma_{n}^{z}\right] .
$$

Now introducing the Pauli matrices

$$
\sigma_{n}^{x}=\sigma_{n}^{+}+\sigma_{n}^{-}, \quad \sigma_{n}^{y}=-i\left(\sigma_{n}^{+}-\sigma_{n}^{-}\right),
$$

the above Hamiltonian reads

$$
\mathcal{H}=\frac{1}{2} \sum_{n}\left[-\kappa_{n}\left(\sigma_{n}^{x} \sigma_{n-1}^{x}+\sigma_{n}^{y} \sigma_{n-1}^{y}\right)+\mu \sigma_{n}^{z}\right] .
$$

This is nothing but the isotropic $X Y$ model with sitedependent hopping.

\section{B. Experimental simulation}

The Bose-Hubbard model in Eq. (14) can be realized in laboratory with various systems for implementing quantum simulation, such as optical lattices, superconducting qubits, and trapped ions. Here we just concentrate on a simple case, which consists of a linear chain of ions in a linear Paul trap. In a linear trap, ions are arranged in a Coulomb chain. Assuming $x$ as one of the transverse directions and $z$ the trap axis, the Hamiltonian of the chain with $N$ ions is $H=V_{0}+V_{C}+V_{L}$, where

$$
\begin{gathered}
V_{0}=\frac{1}{2} m \sum_{i=1}^{N}\left(\omega_{x}^{2} x_{i}^{2}+\omega_{y}^{2} y_{i}^{2}+\omega_{z}^{2} z_{i}^{2}\right), \\
V_{C}=\sum_{i>j}^{N} \frac{e^{2}}{\sqrt{\left(z_{i}-z_{j}\right)^{2}+\left(x_{i}-x_{j}\right)^{2}+\left(y_{i}-y_{j}\right)^{2}}}, \\
V_{L}=\sum_{j>i}^{N} t_{i, j}\left(a_{i}^{\dagger} a_{j}+a_{i} a_{j}^{\dagger}\right),
\end{gathered}
$$

where $\omega_{\alpha}, \alpha=x, y, z$ are the trapping frequencies in each direction, $V_{C}$ is the Coulomb energy, while $V_{L}$ is the coupling between different axial modes, and $t_{i, j}$ are the hopping energies that are induced by a pair of Raman lasers. For a linear trap $\omega_{x, y} \gg \omega_{z}$, the ions form a chain along the $z$ axis and occupy equilibrium positions. Phonons in the $z$ direction can be described approximately by [41]

$$
H=\sum_{i=1}^{N} \omega_{x} a_{i}^{\dagger} a_{i}+\sum_{j>i}^{N} t_{i, j}\left(a_{i}^{\dagger} a_{j}+a_{i} a_{j}^{\dagger}\right) .
$$

Note that $t_{i, j}$ can be precisely adjusted to being site (mode) dependent by varying the phase and the detuning of the Raman beams. By this scheme, we use the phonon modes of trapped ions to realize the Bose-Hubbard model with zero on-site energy. To simulate $n$-site Hubbard modes, we need to trap $N$ ions in a linear trap and use $N-1$ pairs of lasers to drive photon transitions between the $N$ axial modes.

\section{APPLICATIONS IN BLACK-HOLE PHYSICS}

\section{A. Hawking radiation and its entanglements}

In this section, we use the above quantum many-body model to study quantum aspects of black holes in gravity. Let us consider the bosonic hopping model as an example. For convenience in numerical computations, let us specify the function $f(x)=\alpha \tanh x$ and $d=0.1$. In this case, there is a horizon at $x=x_{h}=0$ with the Hawking temperature $T_{H}=\alpha /(4 \pi)$. It is worth noting that $\kappa_{n} \neq 0$ at the horizon, though $f(x)$ is zero at the horizon. Without loss of generality, we set $\mu=0$ as the total particle number is conserved.

To study the black-hole evaporation, we set a particle in the inner region of the black hole by initial state $|\Psi(0)\rangle=\left|e_{n_{0}}\right\rangle$ 

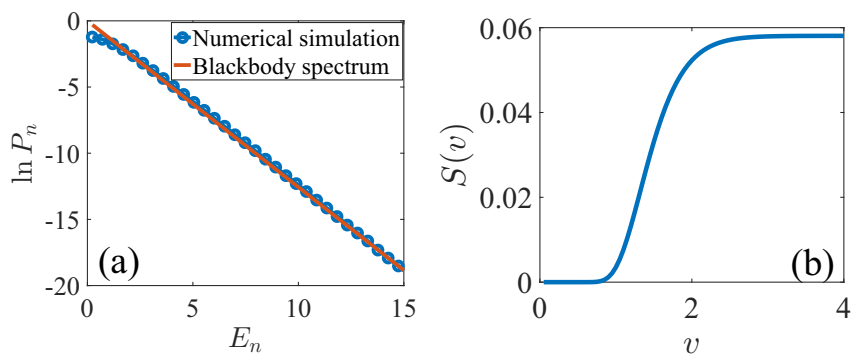

FIG. 1. (a) Numerical simulation on the probability of finding a particle with energy $E_{n}$ in the outer region at early time $v=4 \lesssim$ $O(2 d L / \alpha)$. (b) The evolution of entanglement entropy between the inner region and outer region. The blackbody spectrum is given by Eq. (23). The low-energy limit requires that $E_{n} \ll O(\alpha / d)$.

and choose $n_{0}=-2 / d$ as an example. It describes an initial particle which is localized at the $n_{0}$ th site. Based on the picture of "pair creation" in Hawking radiation, "particle-antiparticle pairs" can be created around the horizon. The antiparticle (negative energy) falls into the black hole and annihilates with this particle inside the black hole, and the particle outside the horizon is materialized and escapes into infinity. Note that the pair creation and annihilation is a virtual process, and the real materialized result is that the original particle inside the black hole disappears but an identical particle appears outside the horizon. This leads to an equivalent picture to understand Hawking radiation via quantum tunneling: the particle inside the horizon escapes to the outside by quantum tunneling. According to Refs. [42-44], neglecting the back-reaction of the radiation, the probability of finding this particle outside the horizon and its energy should obey the following blackbody spectrum:

$$
P(E) \propto e^{-E / T_{H}} .
$$

In Fig. 1 we show the numerical results about the probability of finding a particle of energy $E_{n}$ in the outer region. Here $E_{n}$ is the positive eigenvalue of the Hamiltonian for the outer subregion. The evolutional time is chosen so that the radiation does not touch the cutoff boundary. For the details of numerical calculation, one may refer to Appendix B. We see that numerical results show that $P(E)$ satisfies the blackbody spectrum, Eq. (23), approximately with the temperature $T=T_{H}$. Note that the numerical results for smaller energy deviate from the blackbody spectrum (23), because our finite-size cutoff cannot cover the low-energy region with $E \lesssim O(2 \pi \alpha /(L d))$ and so leads to the deviation. In addition, we also compute the entanglement entropy between the inner region and the outer region, which is given by $S(v)=-\operatorname{Tr}[\rho(v) \ln \rho(v)]$ and the reduced density matrix for the outer region is given by $\rho(v)=\operatorname{Tr}_{\text {inner }}(|\Psi(v)\rangle\langle|\Psi(v)|)$. It shows that entanglement between the inner and outer regions increases during the Hawking radiation. Because there is only one particle in the black hole, the evaporation will stop in a short time and so the entanglement entropy saturates.

\section{B. Quantum chaos and fastest scrambling}

In this section, let us exhibit how to use our analog model to study some new features of quantum field theory in curved spacetime: quantum chaos and fastest scrambling of black holes, appearing from the AdS-CFT correspondence. To supply an asymptotic $\mathrm{AdS}_{2}$ black hole background, we consider $f(x)=x^{2}\left(1-x_{h} / x\right)$ as an example.

To describe the quantum chaos, it was proposed recently that the "out-time order correlation" (OTOC) may serve as a useful characteristic of quantum-chaotic behavior. For two local operators $\hat{W}(t)$ and $\hat{V}(t)$ in the Heisenberg picture, their OTOC is typically defined as

$$
C(t):=-\langle[\hat{W}(t), \hat{V}(0)]\rangle .
$$

Here $\hat{W}(0)$ and $\hat{V}(0)$ can be the same or different; $\langle\cdot\rangle$ stands for average in an initial state. Reference [30] shows that, with a few general assumptions on the underlying field model and in thermal equilibrium state, the growth of a general OTOC $C(t)$ satisfies

$$
C(t) \propto e^{\lambda_{L} t},
$$

where $\lambda_{L}$ is the Lyapunov exponent and satisfies the following "chaos bound":

$$
\lambda_{L} \leqslant 2 \pi T .
$$

Here $T$ is the temperature of the system. The exponential growth (25) will be broken after the "scrambling time"

$$
t_{*} \geqslant \frac{1}{2 \pi T} \ln N_{f}^{2}, \quad N_{f}^{2} \gg 1 .
$$

Here $N_{f}$ stands for the degrees of freedom of the system. It is conjectured in Refs. [28-30] that a black hole is the most chaotic system and has the fastest scrambling; i.e., it saturates the bounds (26) and (27).

Now let us employ our model to check if it can exhibit the exponential growth of OTOC and give us a positive Lyapunov exponent. As an example, we numerically study the following OTOC:

$$
C(v):=-\operatorname{Tr}\left(\rho\left[\hat{N}_{n_{0}}(v), \hat{N}_{n_{0}}\right]^{2}\right) .
$$

Here $\hat{N}_{n_{0}}$ is a local operator associated to the particle number operator at the $n_{0}$ th site,

$$
\hat{N}_{n_{0}}=\frac{d}{l_{0}} \sum_{n=-L}^{L} \hat{a}_{n}^{\dagger} \hat{a}_{n} e^{-d^{2}\left(n-n_{0}\right)^{2} / l_{0}^{2}} .
$$

Here $l_{0}$ is the length scale and stands for the width of distribution of $\hat{N}_{n_{0}}$. The time-evolution operator $\hat{N}_{n}$ is given by the Heisenberg picture $\hat{N}_{n}(v)=\exp (-i \mathcal{H} v) \hat{N}_{n} \exp (i \mathcal{H} v)$. The reason we use Eq. (29) to define the local operator $\hat{N}_{n_{0}}$ rather than $\hat{N}_{n_{0}}=\hat{a}_{n_{0}}^{\dagger} \hat{a}_{n_{0}}$ is that Eq. (29) is a well-defined smooth local operator in the continuous limit $d \rightarrow 0$. Instead, $\hat{N}_{n_{0}}=\hat{a}_{n_{0}}^{\dagger} \hat{a}_{n_{0}}$ will become a $\delta$-like function in the continuous limit, which is singular. The initial state is a thermal state with the temperature the same as the temperature of the black hole:

$$
\rho=\frac{1}{Z} \sum_{E_{\text {out }}} e^{-\beta E_{\text {out }}}\left|E_{\text {out }}\right\rangle\left\langle E_{\text {out }}\right| .
$$

Here $Z$ is the normalized factor which ensures $\operatorname{Tr}(\rho)=1$ and the summation contains all the positive energy modes of the outside Hamiltonian $\mathcal{H}_{\text {out }}$ (as the negative modes are assumed to fall into the black hole). $\mathcal{H}_{\text {out }}$ is obtained by only extracting the sites outside the horizon in Eqs. (15), (16), and (18). 


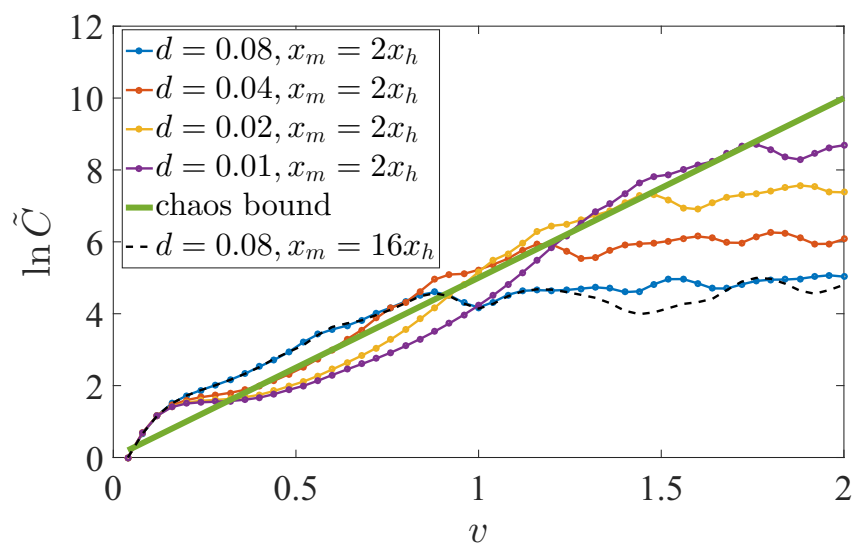

FIG. 2. The numerical simulations on evolution of OTOC when $l_{0}=x_{h} / 15, n_{0}=\left(x_{h}+2 l_{0}\right) / d$. The solid green line is fitted based on Eq. (25), where the Lyapunov exponent $\lambda_{L}=2 \pi T_{H}$. Here we choose $x_{h}=10$. We numerically simulate the results for time $v=0.02 \sim 2$ and $d=0.01,0.02,0.04$, and 0.08. $x_{m}$ is the position of the AdS boundary. The numerical results are not sensitive to the value of the cutoff boundary in the region of exponential growth.

The time evolution of $C(v)$ is obtained numerically. The results are shown in Fig. 2. For convenience, we define $\tilde{C}(v)=C(v) / C(0.02)$, which does not change the slope of $\ln C(v)$. Some details of numerical simulations are shown in Appendix C. We can observe that $C(v)$ exponentially grows approximately in the early time. The slope of the fitting curve is found to be $2 \pi T_{H}$ approximately. The chaos bound (26) is saturated approximately. In the pure gravity theory, the effective degree of freedom will be proportional to $G^{-2}$ [30], where $G$ is Newton's constant. Here we neglect the back-reaction of matters on geometry, which means the limit of $G \rightarrow 0$. Thus, in principle, the OTOC will increase forever, i.e., $t_{*} \rightarrow \infty$. However, as we here use the lattice model, the operators and their commutators are bounded and so exponential growth will stop at a finite time. We study how the $C(v)$ depends on the discrete distance $d$. The results show that the time scale of exponential growth will increase if we decrease $d$ but fix the horizon radius $x_{h}$ and distribution width $l_{0}$ of $\hat{N}_{n_{0}}$. This suggests that the time scale of exponential growth will become infinity in the continuous limit $d \rightarrow 0$, as expected.

Strictly speaking, to claim a system to be chaotic, either classically or quantum mechanically, the positive Lyapunov exponent is necessary but not sufficient. The positive Lyapunov exponent only indicates the sensitivity to the initial perturbations, which is the necessary condition of chaos. For example, in the classical case, we also require that the trajectory is dense in a neighborhood of phase space (i.e., ergodic). However, the linear analysis is enough to help us to find the Lyapunov exponents in both the classical case and the quantummechanical case. This can be understood by recalling the standard method in computing the Lyapunov exponent of classical chaotic systems. Thus, a linearized theory in a black-hole background is enough to check the "chaos bound" (it may be more suitable to call it the "bound on Lyapunov exponent").

In order to check if models (15), (16), and (18) really contain chaotic behaviors when the coupling constants are given by a black-hole metric, we study the statistics of

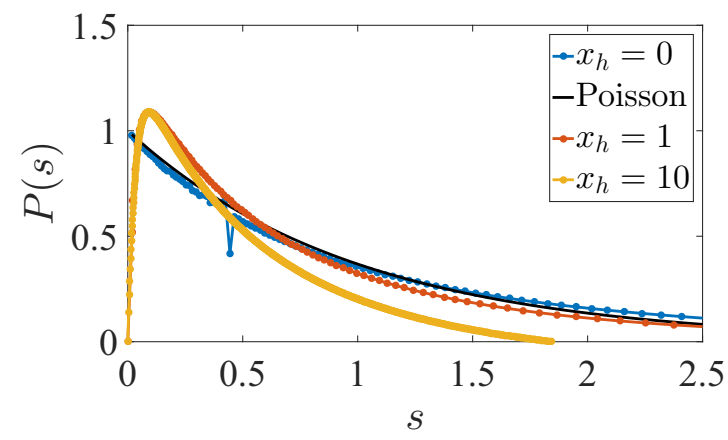

FIG. 3. Distribution of nearest-neighbor level spacings when the hopping $\kappa_{n}$ is given by a pure AdS spacetime (blue) and black holes (red and yellow), respectively. The black line is given by the Poisson distribution $P(s)=e^{-s}$. We choose $d=0.02$ for all cases, $\left\{x_{h}=0, x_{m}=10\right\}$ for the pure AdS case, and $\left\{x_{h}=1,10, x_{m}=5 x_{h}\right\}$ for the black-hole cases. Here $x_{m}$ is the cutoff AdS boundary. An integrable-nonintegrable phase transition occurs when $x_{n} \neq 0$.

"nearest-neighbor level spacing," which is an other characteristic quantity of chaotic systems. We denote the energy levels of the outside Hamiltonian to be $E_{i}$ with $E_{i}<E_{i+1}$, which are obtained by directly diagonalizing the Hamiltonian numerically (the cutoff in high energy levels is needed as high energy levels have low accuracy and are not trustworthy in physics). We assume that $\Delta$ is the mean value of $E_{i+1}-E_{i}$ and $\mathcal{N}$ is the total number of energy levels. Then we define $\mathcal{N} P(s) \delta s$ to be the number of energy levels $E_{i}$ which satisfy $s \leqslant\left(E_{i+1}-E_{i}\right) / \Delta \leqslant s+\delta s$. The function $P(s)$ is called the "nearest-neighbor level spacing" function. It has been shown that if the system is integrable, $P(s)$ satisfies Poisson statistics $P(s)=e^{-s}$ [45]. If the system is chaotic, $P(s)$ will deviate from the Poisson statistics. For a Gaussian orthogonal ensemble or Gaussian unitary ensemble, $P(s)$ is given by a Wigner distribution. For other general cases, $P(s)$ may be given by a general Brody distribution approximately [46]. In Fig. 3 we show the numerical results about $P(s)$. For the case that $x_{h}=$ 0 , the effective spacetime has no black hole and we find that $P(s)$ is given by a Poisson statistics approximately. However, once $x_{h} \neq 0$, we find that a dramatic change happens and $P(s)$ is no longer a Poisson distribution, which suggests that the system is not integrable.

From the viewpoint of quantum many-body theory, Hamiltonians shown in Eqs. (14), (15), and (18) contain only nearest-neighbor hopping and quadratic interactions do not matter in the design of coupling constants. When we design the coupling constant by setting $x_{h} \neq 0$, it is not easy to understand why these models can exhibit exponential growth of OTOC and why the systems have an "integrable-nonintegrable phase transition." However, if we use our framework to convert them into an effective black hole, we immediately understand these properties. When $x_{h} \neq 0$, a black-hole metric is encoded into the site-dependent coupling $\kappa_{n}$ and so the system has exponential growth of OTOC and a signal of chaos. This offers an example about how to use our models to get some insights about the quantum many-body systems from black-hole physics. 


\section{SUMMARY}

In summary, we have shown that a massless scalar or Dirac field in the static $(1+1)$-dimensional curved spacetime can be simulated by some basic models in condensed-matter physics: the bosonic hopping model, the free Hubbard model, and the $X Y$ model. We suggested a possible experimental realization in trapped-ion systems for this analog gravity model. As some applications of the analog gravity model, we have numerically shown that this model can be used to simulate Hawking radiation. We have also checked the quantum chaos behavior of black holes and verified that a black hole is one of the most chaotic systems and has the fastest scrambling in nature. These are predictions of AdS-CFT correspondence. In this sense our model provides the possibility to test experimentally the correspondence. In addition, our results show that the site-dependent hopping has a one-to-one relation to the spacetime point of a curved background. By a concrete example, we showed how this framework can help us get some insights about the quantum many-body systems from blackhole physics. This not only provides new motivation to study the site-dependent hopping model, but also indicates a large number of applications in the analog gravity model. These will bring us new viewpoints and phenomena of quantum many-body systems, and also will enlighten us to deeply understand the nature of gravity.

\section{ACKNOWLEDGMENTS}

R.-G.C. was supported in part by the National Natural Science Foundation of China, Grants No. 11690022, No. 11821505, No. 11851302, No. 11947302, and No. 11745006, by the Strategic Priority Research Program of CAS, Grant No. XDB23030100, and by the Key Research Program of Frontier Sciences of CAS. H.L. was supported by the National Natural Science Foundation of China, Grant No. 11690033. L.L. is a member of the Indiana University Center for Spacetime Symmetries (IUCSS). L.L. received support from the National Natural Science Foundation of China under Grant No. 11774436, Sun Yat-sen University Discipline Construction Fund, Sun Yat-sen University Three Major Construction Fund, and Guangdong Province Pearl River Youth Talent Fund.

\section{APPENDIX A: TUNNELING RATE AND HAWKING TEMPERATURE}

In this Appendix, we show how to use the picture of "quantum tunneling" to obtain the tunneling rate and the Hawking temperature of a black hole.

To obtain the tunneling rate, we need to find the solution of Eq. (8) with the energy $E$. By a variable transformation

$$
w=\phi / \sqrt{f}
$$

we can find that

$$
\partial_{v} \phi=-\frac{f}{2} \partial_{x} \phi
$$

The positive energy (measured by $v$ ) solution is

$$
\phi=\phi_{0} \exp \left[-i E\left(v-2 \int \frac{d x}{f(x)}\right)\right] \text {. }
$$

As $f(x)=0$ at the horizon, this solution is not continuous at the horizon. Let us separate the integration in the above equation as follows:

$$
\begin{aligned}
\int \frac{d x}{f(x)} & =\int\left(\frac{1}{f(x)}-\frac{1}{2 g_{h}\left(x-x_{h}\right)}\right) d x+\frac{1}{2 g_{h}} \ln \left|x-x_{h}\right| \\
& =F(x)+\frac{1}{2 g_{h}} \ln \left|x-x_{h}\right| .
\end{aligned}
$$

The function $F(x)$ is continuous at the horizon. The divergence has been absorbed into the logarithmic function. The solution (A3) can be separated into two pieces,

$$
\phi=\phi_{1} \exp \left\{-i E\left[v-2 F(x)-\frac{1}{g_{h}} \ln \left(x_{h}-x\right)\right]\right\}
$$

for $x<x_{h}$ and

$$
\phi=\phi_{2} \exp \left\{-i E\left[v-2 F(x)-\frac{1}{g_{h}} \ln \left(x-x_{h}\right)\right]\right\}
$$

for $x>x_{h}$. The tunneling rate then reads

$$
\Gamma:=\frac{\left|\phi_{2}\right|^{2}}{\left|\phi_{1}\right|^{2}} \text {. }
$$

Following the argument in Ref. [42], the two pieces of the solution in Eqs. (A5) and (A6) should be connected continuously under the bottom half of the complex plane. Treating the piece of $x<x_{h}$ as the starting point and analytically continuing it into the region of $x>x_{h}$, the logarithmic function in Eq. (A5) will obtain an additional phase factor and so we can obtain the following relationship:

$$
\phi_{1} \exp \left(-\frac{\pi E}{g_{h}}\right)=\phi_{2} .
$$

Taking it into Eq. (A7), we then obtain

$$
\Gamma=\exp \left(-\frac{2 \pi E}{g_{h}}\right)=\exp \left(-\frac{E}{T_{H}}\right) .
$$

As expected, the tunneling rate and energy satisfy the blackbody spectrum, and the temperature is just given by $T_{H}=$ $g_{h} /(2 \pi)$.

In physics, Eq. (A3) implies an infinite momentum at the horizon and so we break down our condition for discretization. This belongs to the question of the "trans-Planckian problem," which widely exists in all discussions of Hawking radiation. A particle emitted from a black hole with a finite frequency (measured at infinity), if traced back to the horizon, must have an infinite momentum, and therefore a trans-Planckian wavelength. The trans-Planckian problem is a mathematical artifact of horizon calculations. In all analog models, when the emitted particle is near the "horizon," the smooth approximation is invalid and so a truncation is needed. However, it has been shown that the details of truncation will not change the behavior of Hawking radiation in the low-energy region (where "low energy" means the energy is low at infinity; see Ref. [4], for example). 


\section{APPENDIX B: DETAILS OF NUMERICAL SIMULATIONS ON HAWKING RADIATION}

Let us first explain how to make the numerical simulation on Hawking radiation. We take $f(x)=\alpha \tanh x$. Then we can see that the hopping amplitude reads

$$
\kappa_{n}=\frac{\alpha \tanh [(n-1 / 2) d]}{4 d} .
$$

There is a horizon at $x=x_{h}=0$ with the Hawking temperature $T_{H}=\alpha /(4 \pi)$. It is worth noting that $\kappa_{n} \neq 0$ at the horizon though $f(x)$ is zero at the horizon. The numerical computation needs a finite cutoff $n=-L,-L+1, \ldots, L-1, L$. To match this cutoff, we have to set the hopping amplitude $\kappa_{n}$ such that

$$
\kappa_{n}=0, \quad \text { if } n \geqslant L \text { or } n \leqslant-L .
$$

Without loss of generality, we set $\mu=0$ as the total particle number is conserved.

The Hamiltonians for the inner region and outer region are

$$
\mathcal{H}_{\text {in }}=-\sum_{n=-L}^{-1} \kappa_{n}\left(\hat{a}_{n}^{\dagger} \hat{a}_{n-1}+\hat{a}_{n-1}^{\dagger} \hat{a}_{n}\right)
$$

and

$$
\mathcal{H}_{\text {out }}=-\sum_{n=2}^{L} \kappa_{n}\left(\hat{a}_{n}^{\dagger} \hat{a}_{n-1}+\hat{a}_{n-1}^{\dagger} \hat{a}_{n}\right) .
$$

Note that the total Hamiltonian is not the sum of the inner part and the outer part. In fact, we have

$$
\mathcal{H}=\mathcal{H}_{\text {in }}+\mathcal{H}_{\text {out }}+\mathcal{H}_{0} \text {. }
$$

Here $\mathcal{H}_{0}$ is the contribution at the horizon,

$$
\mathcal{H}_{0}=-\kappa_{0}\left(\hat{a}_{0}^{\dagger} \hat{a}_{-1}+\hat{a}_{-1}^{\dagger} \hat{a}_{0}\right)-\kappa_{1}\left(\hat{a}_{1}^{\dagger} \hat{a}_{0}+\hat{a}_{0}^{\dagger} \hat{a}_{1}\right),
$$

which mixes the inner region and outer region.

Assume $N$ to be the total particle number. It is difficult to simulate the dynamics for large $N$ and $L$. For example, in the case $2 L+1=N=13$, the dimension of the total Hilbert space is $D \approx 5 \times 10^{6}$. To simplify the issue in a numerical algorithm, let us choose $N=1$ and so the dimension of the Hilbert space is $D=2 L+1$. In this case, we can choose the eigenvectors of $\hat{a}_{n}^{\dagger} \hat{a}_{n}$ as the basic vectors of the Hilbert space,

$$
\begin{aligned}
& \left|e_{-L}\right\rangle=(1,0, \ldots, 0)^{T}, \\
& \left|e_{-L+1}\right\rangle=(0,1,0, \ldots, 0)^{T}, \\
& \ldots, \\
& \left|e_{L}\right\rangle=(0,0,0, \ldots, 1)^{T},
\end{aligned}
$$

which satisfy

$$
\left\langle e_{l}\left|\hat{a}_{n}^{\dagger} \hat{a}_{n}\right| e_{k}\right\rangle=\delta_{n l} \delta_{n k}
$$

and

$$
\left\langle e_{l}\left|\hat{a}_{n}^{\dagger} \hat{a}_{n-1}\right| e_{k}\right\rangle=\delta_{n-1, k} \delta_{l, n} .
$$

Then we can write the matrix elements of the Hamiltonian:

$$
\begin{aligned}
& \left(\mathcal{H}_{\text {in }}\right)_{l, k}= \begin{cases}-\kappa_{l}\left(\delta_{k, l-1}+\delta_{l, k-1}\right), & k, l \leqslant-1 \\
0, & k, l>-1,\end{cases} \\
& \left(\mathcal{H}_{\text {out }}\right)_{l, k}= \begin{cases}-\kappa_{l}\left(\delta_{k, l-1}+\delta_{l, k-1}\right), & k, l \geqslant 2 \\
0, & k, l<2,\end{cases}
\end{aligned}
$$

and

$$
(\mathcal{H})_{l, k}=-\kappa_{l}\left(\delta_{k, l-1}+\delta_{l, k-1}\right) .
$$

For a given initial state $|\Psi(0)\rangle$, the time-evolution state is given by $|\Psi(v)\rangle=e^{-i \mathcal{H} v}|\Psi(0)\rangle$. In Fig. 1, we take parameters $d=0.1, L=300$, and $\alpha=10$. The results are similar if we choose $N=2$. Due to the technical difficulties addressed above, we cannot explore larger $N$. Roughly speaking, as we consider the free theory in fixed background, many particles can be understood as a collection of single particles. Thus, the simplification here does not lose the essential physics.

\section{APPENDIX C: DETAILS OF NUMERICAL SIMULATIONS ON OTOC}

The simulation on OTOC is similar. We take $f(x)=$ $x^{2}\left(1-x_{h} / x\right)$. Then we can see that the hopping reads

$$
\kappa_{n}=\frac{f[(n-1 / 2) d]}{4 d} .
$$

There is a horizon at $x=x_{h}$ with the Hawking temperature $T_{H}=x_{h} /(4 \pi)$. In this case we make the cutoff in the following way:

$$
n=1,2, \ldots, 2 L+1, \quad \text { with } L d=x_{h} .
$$

Similar to the case in Hawking radiation, if we choose $N=$ 1, we still have Eqs. (B6) and (B7). Then we can write the matrix elements of the Hamiltonian

$$
(\mathcal{H})_{l, k}=-\kappa_{l}\left(\delta_{k, l-1}+\delta_{l, k-1}\right)
$$

and

$$
\left(\hat{a}_{n}^{\dagger} \hat{a}_{n}\right)_{k l}=\delta_{n l} \delta_{n k} .
$$

For a given initial state $|\Psi(0)\rangle$, the time-evolution state is given by $|\Psi(v)\rangle=e^{-i \mathcal{H} v}|\Psi(0)\rangle$ and $\hat{N}_{n}(v)=$ $\exp (-i \mathcal{H} v) \hat{N}_{n} \exp (i \mathcal{H} v)$. Then we can obtain the OTOC in Eq. (27).
[1] T. Jacobson, Introduction to quantum fields in curved spacetime and the Hawking effect, in Lectures on Quantum Gravity, edited by A. Gomberoff and D. Marolf (Springer, Boston, MA, 2005), pp. 39-89.
[2] S. Hollands and R. M. Wald, Quantum fields in curved spacetime, Phys. Rep. 574, 1 (2015).

[3] W. G. Unruh, Experimental Black-Hole Evaporation? Phys. Rev. Lett. 46, 1351 (1981). 
[4] W. G. Unruh, Sonic analog of black holes and the effects of high frequencies on black hole evaporation, Phys. Rev. D 51, 2827 (1995)

[5] S. Weinfurtner, E. W. Tedford, M. C. J. Penrice, W. G. Unruh, and G. A. Lawrence, Measurement of Stimulated Hawking Emission in an Analogue System, Phys. Rev. Lett. 106, 021302 (2011).

[6] L. J. Garay, J. R. Anglin, J. I. Cirac, and P. Zoller, Sonic Analog of Gravitational Black Holes in Bose-Einstein Condensates, Phys. Rev. Lett. 85, 4643 (2000).

[7] J. Steinhauer, Observation of quantum Hawking radiation and its entanglement in an analogue black hole, Nat. Phys. 12, 959 (2016).

[8] J. Ramón Muñoz de Nova, K. Golubkov, V. I. Kolobov, and J. Steinhauer, Observation of thermal Hawking radiation and its temperature in an analogue black hole, Nature (London) 569, 688 (2019).

[9] C. Sheng, H. Liu, Y. Wang, S. N. Zhu, and D. A. Genov, Trapping light by mimicking gravitational lensing, Nat. Photonics 7 , 902 (2013).

[10] R. Bekenstein, Y. Kabessa, Y. Sharabi, O. Tal, N. Engheta, G. Eisenstein, A. J. Agranat, and M. Segev, Control of light by curved space in nanophotonic structures, Nat. Photonics 11, 664 (2017).

[11] J. Drori, Y. Rosenberg, D. Bermudez, Y. Silberberg, and U. Leonhardt, Observation of Stimulated Hawking Radiation in an Optical Analogue, Phys. Rev. Lett. 122, 010404 (2019).

[12] C. Sheng, H. Liu, H. Chen, and S. Zhu, Definite photon deflections of topological defects in metasurfaces and symmetrybreaking phase transitions with material loss, Nat. Commun. 9, 4271 (2018).

[13] F. Zhong, J. Li, H. Liu, and S. Zhu, Controlling Surface Plasmons Through Covariant Transformation of the SpinDependent Geometric Phase Between Curved Metamaterials, Phys. Rev. Lett. 120, 243901 (2018).

[14] J. S. Pedernales, M. Beau, S. M. Pittman, I. L. Egusquiza, L. Lamata, E. Solano, and A. del Campo, Dirac Equation in (1+1)Dimensional Curved Spacetime and the Multiphoton Quantum Rabi Model, Phys. Rev. Lett. 120, 160403 (2018).

[15] C. Barcelo, S. Liberati, and M. Visser, Analogue gravity, Living Rev. Relativ. 8, 12 (2005); Analogue gravity, 14, 3 (2011)

[16] D. Faccio, F. Belgiorno, S. Cacciatori, V. Gorini, S. Liberati, and U. Moschella, Analogue Gravity Phenomenology (Springer International Publishing, Cham, Switzerland, 2013).

[17] C. Barceló, Analogue black-hole horizons, Nat. Phys. 15, 210 (2018).

[18] H. Tasaki, The Hubbard model - an introduction and selected rigorous results, J. Phys.: Condens. Matter 10, 4353 (1998).

[19] J. Hu, L. Feng, Z. Zhang, and C. Chin, Quantum simulation of Unruh radiation, Nat. Phys. 15, 785 (2019).

[20] N. Trautmann and P. Hauke, Quantum simulation of the dynamical Casimir effect with trapped ions, New J. Phys. 18, 043029 (2016).

[21] R. Schutzhold and W. G. Unruh, Cosmological particle creation in the lab? In Analogue Gravity Phenomenology, Lecture Notes in Physics Vol. 870 (Springer, Cham, 2013), pp. 51-61.

[22] J. M. Maldacena, The large-N limit of superconformal field theories and supergravity, Int. J. Theor. Phys. 38, 1113 (1999).
[23] E. Witten, Anti-de Sitter space and holography, Adv. Theor Math. Phys. 2, 253 (1998).

[24] S. S. Gubser, I. R. Klebanov, and A. M. Polyakov, Gauge theory correlators from noncritical string theory, Phys. Lett. B 428, 105 (1998).

[25] S. Ryu and T. Takayanagi, Holographic Derivation of Entanglement Entropy from the anti-de Sitter Space/Conformal Field Theory Correspondence, Phys. Rev. Lett. 96, 181602 (2006).

[26] T. Nishioka, S. Ryu, and T. Takayanagi, Holographic entanglement entropy: An overview, J. Phys. A 42, 504008 (2009).

[27] P. Hayden and J. Preskill, Black holes as mirrors: Quantum information in random subsystems, J. High Energy Phys. 09 (2007) 120.

[28] S. H. Shenker and D. Stanford, Black holes and the butterfly effect, J. High Energy Phys. 03 (2014) 067.

[29] Y. Sekino and L. Susskind, Fast scramblers, J. High Energy Phys. 10 (2008) 065.

[30] J. Maldacena, S. H. Shenker, and D. Stanford, A bound on chaos, J. High Energy Phys. 08 (2016) 106.

[31] R. B. Mann, S. M. Morsink, A. E. Sikkema, and T. G. Steele, Semiclassical gravity in 1+1 dimensions, Phys. Rev. D 43, 3948 (1991).

[32] H. A. Gersch and G. C. Knollman, Quantum cell model for bosons, Phys. Rev. 129, 959 (1963).

[33] M. Ma, B. I. Halperin, and P. A. Lee, Strongly disordered superfluids: Quantum fluctuations and critical behavior, Phys. Rev. B 34, 3136 (1986).

[34] T. Giamarchi and H. J. Schulz, Anderson localization and interactions in one-dimensional metals, Phys. Rev. B 37, 325 (1988).

[35] M. P. A. Fisher, P. B. Weichman, G. Grinstein, and D. S. Fisher, Boson localization and the superfluid-insulator transition, Phys. Rev. B 40, 546 (1989).

[36] T. Hensgens, T. Fujita, L. Janssen, X. Li, C. J. Van Diepen, C. Reichl, W. Wegscheider, S. Das Sarma, and L. M. K. Vandersypen, Quantum simulation of a Fermi-Hubbard model using a semiconductor quantum dot array, Nature (London) 548, 70 (2017).

[37] L. Tarruell and L. Sanchez-Palencia, Quantum simulation of the Hubbard model with ultracold fermions in optical lattices, C. R. Phys. 19, 365 (2018).

[38] J. Salfi, J. A. Mol, R. Rahman, G. Klimeck, M. Y. Simmons, L. C. L. Hollenberg, and S. Rogge, Quantum simulation of the Hubbard model with dopant atoms in silicon, Nat. Commun. 7, 11342 (2016).

[39] E. Barouch, B. M. McCoy, and M. Dresden, Statistical mechanics of the XY model. I, Phys. Rev. A 2, 1075 (1970).

[40] E. Barouch and B. M. McCoy, Statistical mechanics of the $X Y$ model. II. Spin-correlation functions, Phys. Rev. A 3, 786 (1971).

[41] D. Porras and J. I. Cirac, Bose-Einstein Condensation and Strong-Correlation Behavior of Phonons in Ion Traps, Phys. Rev. Lett. 93, 263602 (2004).

[42] T. Damour and R. Ruffini, Black hole evaporation in the Klein-Sauter-Heisenberg-Euler formalism, Phys. Rev. D 14, 332 (1976). 
[43] M. K. Parikh and F. Wilczek, Hawking Radiation as Tunneling, Phys. Rev. Lett. 85, 5042 (2000).

[44] M. Arzano, A. J. M. Medved, and E. C. Vagenas, Hawking radiation as tunneling through the quantum horizon, J. High Energy Phys. 09 (2005) 037.
[45] T. Guhr, A. Müller-Groeling, and H. A. Weidenmüller, Random-matrix theories in quantum physics: Common concepts, Phys. Rep. 299, 189 (1998).

[46] H. Sabri, Sh. S. Hashemi, B. R. Maleki, and M. A. Jafarizadeh, Generalization of Brody distribution for statistical investigation, Random Matrices: Theory Appl. 03, 1450017 (2014). 